LBL --29776

DE91 005370

\title{
DROP-TOWER EXPERIMENTS FOR CAPILLARY SURFACES IN AN EXOTIC CONTAINER ${ }^{1}$
}

\author{
Paul Concus \\ Lawrence Berkeley Laboratory \\ and \\ Department of Mathematics \\ University of California \\ Berkeley, CA 94720 \\ Robert Finn \\ Department of Mathematics \\ Stanford University \\ Stanford, CA 94305 \\ Mark Weislogel \\ NASA Lewis Research Center \\ Cleveland, $\mathrm{OH} 44135$
}

October 1990

To be presented at
AlAA 29th Acrospace Sciences Meeting
Reno, NV
January $7-10,1991$

DISCLAIMER

\begin{abstract}
This report was prepared as an account of work sponsored by an agency of the United States Guvernment. Neither the United States (iovernment nor any agency thereof, nor any of their employees, makes any warranty, express or implied, or assumes any legal liability or responsibility for the accuracy, completeness, or usefulness of any information, apparatus, product, or process disclosed, or represents that its use would not infringe privately owned rights. Reference herein to any specific commercial product, process, or service by trade name, trademark, manufacturer, or otherwise does not necessarily constitute or imply its endorsement, recommendation, or favoring by the United States Government or any agency thereof. The views and opinions of authors expressed herein do not necessarily state or reflect those of the United States Government or any agency thereof.
\end{abstract}

\footnotetext{
1 This work was supported in part by the Applied Mathematical Sciences subprogram of the Office of Energy Research, U.S. Department of Energy under Contract DE-AC03-76SF00098, by the National Aeronautics and Space Administration under Grant NAG3-1143, and by the National Science Foundation under Grant DMS89-02831.
} 


\title{
DROP-TOWER EXPERIMENTS FOR CAPILLARY SURFACES IN AN EXOTIC CONTAINER
}

\author{
Paul Concus* \\ University of California, Berkeley, CA 94720 \\ Robert Finn $\dagger$ \\ Stanford University, Stanford, CA 94305 \\ Mark Weislogel $\ddagger$ \\ NASA Lewis Research Center, Cleveland, OH 44135
}

\begin{abstract}
Low-gravity drop-tower experiments are carried out for an "exotic" rotationally-symmetric container, which admits an entire continuum of distinct equilibrium symmetric capillary free surfaces. It is found that an initial equilibrium planar interface, a member of the continuum, will reorient toward a non-symmetric interface, as predicted by recent mathematical theory.
\end{abstract}

\section{Introduction}

Generally, the free surface of a liquid that partly fills a container under the action of surface and gravitat' nal forces may assume one of several possible equilibrium configurations. It can occur that only one configuration is possible; that happens, for example, in a vertical homogeneous cylindrical container in a gravity field that is either absent or directed downward into the liquid. Whenever the contact curve lies entirely on the cylindrical walls, the free surface is completely determined by the contact angle and liquid volume; this is the case for cylinders of arbitrary cross section. However, examples are easily given of containers for which two or more distinct equilibrium configurations can be found.

In earlier papers,7 we discuss "exotic" rotationally symmetric containers that admit an entire continuum of distinct, rotationally-symmetric equilibrium capillary free-surfaces, all enclosing the same liquid volume and having the same mechanical energy and contact angle. These families of symmetric freesirfaces have the further property that they are unstable, in that certain asymmetric deformations yield surfaces with lower energy. ${ }^{3,7}$ In fact, the containers can be so designed that the only energy-minimizing liquid configurations they admit are not symmetric. Although the theory predicts exotic containers for any gravity field, only for microgravity conditions would the length scale be adequately large for accurate

* Senior Scientist, Lawrence Berkeley Laboratory and Adjunct Professor, Department of Mathematics

$\dagger$ Professor, Department of Mathematics

† Aerospace Engineer, Space Experiments Division physical observation and measurement.

In Ref. 4 these exotic container shapes are calculated by numerical integration of the governing system of nested differential equations, which arise from the classical Young-Laplace equations for equilibrium capillary free surfaces and the condition of prescribed enclosed fluid volume. The shapes are depicted graphically there for a range of gravity accelerations and contact angles of physical interest, along with members of the symmetric equilibrium free-surface continua.

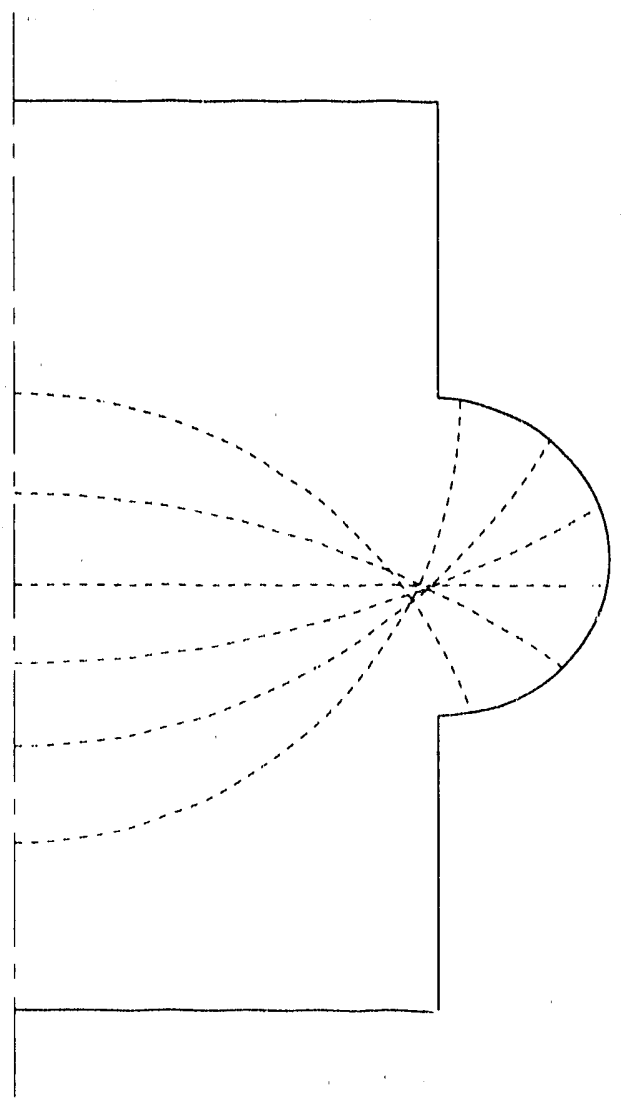

Figure 1. Radial section of an exotic container for contact angle $80^{\circ}$ and zero gravity, depicting meridians (dashed curves) of members of the symmetric equilibrium free surface continuum. 
Here we report on ground-based drop-tower experiments that have been carried out for a particular exotic container, whose radial section is depicted in Fig. 1. This container is for zero gravity and for a contact angle of 80 degrees. The container meridian is shown as the solid curve, and the dashed curves (circular arcs for zero gravity) are meridians of some members of the corresponding continuum of symmetric equilibrium free surfaces. The "exotic", bulge portion of the container has been joined top and bottom for this example hy right circular cylindrical extensions and disk ends.

Of particular interest for experiments is the property noted above, that a configuration of lower meclanical energy cas be obtained by a non-rotationallysymmetric perturbation of the planar member of the family of solution surfaces. Thus, under the idealized Young-Laplace equilibrium contact-angle conditions, if surface friction effects were absent, the family of symmetric equilibrium free surfaces indicated in Fig. 1 would not be observed physically in the container. Through physical experiments we hope to gain an understanding of how a real Huid behaves and to which asymmetric configurations, if any, the fluid might move. The drop-tower experiments reported here are preliminary studies in preparation for a space experiment on the NASA United States Microgravity Laboratory flight (USMI-1) scheduled for 1992.

\section{Container protile calculation}

Consider tha free surface of a liquid that partly fills a rotationally symmetric container oriented with its axis of symmetry parallel to a uniform downwardacting gravitational field, if present. Then a rotationally symmetric equilibrium free-surface of the liquid satisfies

$$
\frac{1}{r} \frac{d}{d r}(r \sin \psi)=B u+\lambda
$$

where $r$ is the radial cuordinate, $u$ is the height of the surface, $\psi$ is the angle between the horizontal and a meridian of he surface, $B$ is the Bond number $(B \geq 0)$, and $\lambda$ is a parameter that is determined by the geometry and volume constraint. ${ }^{\circ}$ The fiee surface is to meet the container in a prescribed contact angle $\gamma, 0<\gamma<\pi$, mecssurec' within the liquid (see Fig. 2).

The container shapes art determined from the requirement that there be a continuum of rotationally symmetric interfaces obtained from the solutions of (1), all having the same contact angle, and enclosing the same volume of liquid with the container. If the container meridian is described parametrically by $r=R(\phi), z=Z(\phi)$, where $\phi$ is the angle with the

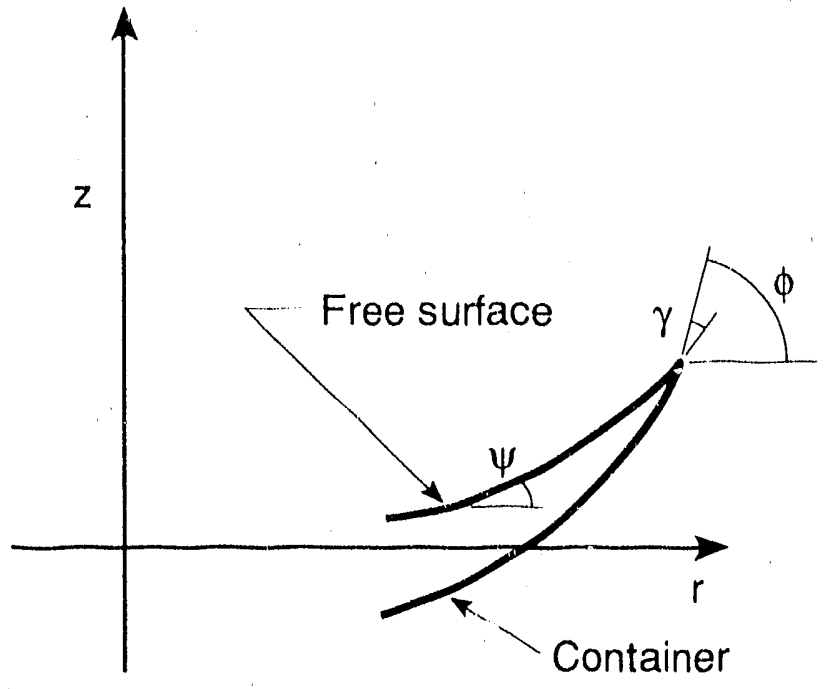

Figure 2. Coordinates for container and free surface calculations.

horizontal (see Fig. 2), then $R$ and $Z$ satisfy

$$
\begin{aligned}
& \frac{d R}{d \phi}=\frac{\cos \phi}{k_{c}}, \\
& \frac{d Z}{d \phi}=\frac{\sin \phi}{k_{c}} .
\end{aligned}
$$

Here $k_{c}$ is the meridional rurvature of the container and can be given as an expression involving $\phi, \gamma$, and quantities relating to the solutions of (1). ${ }^{4}$ As shown in our earlier paper ${ }^{4}$, the system (2), with appropriate initial conditions, can be integrated numerically to obtain the container shapes. The resulting shape calculated for $B=0, \gamma=80^{\circ}$ is the curved bulge shown in Fig. 1. The upper and lower circular cylindrical extensions in Fig. 1 are of the same radius, corresponding to the lower extension meeting the bulge in a right angle (i. e., where $\phi=0$ ). The upper extension intersects the bulge where $\phi \approx 176^{\circ}$. The $80^{\circ}$ contact angle corresponds to the materials used for the experiment, acrylic plastic and distilled waier.

\section{Experimental apparatus}

The fill apparatus and vessel are depicted schematically in Fig. 3. The fill apparatus consists of a gravity feed tank, a solenoid valve, and a vapor return line. The vessel interior was bored out of a solid rectangular block of acrylic plastic, to limit optical distortion. The block had a cross sertion of $10 \mathrm{~cm} \times 10 \mathrm{~cm}$. The coordinates for the bulgedportion of the cylinder, calculated for a contact angle of $80^{\circ}$ and scaled to have a maximum bulge diameter of $7.9 \mathrm{~cm}$, were fed into a numerically controlled air bearing lathe, which performed the final machining operations. As shown in Fig. 1, the circular cylindrical portions bored out above and below the bulge were 


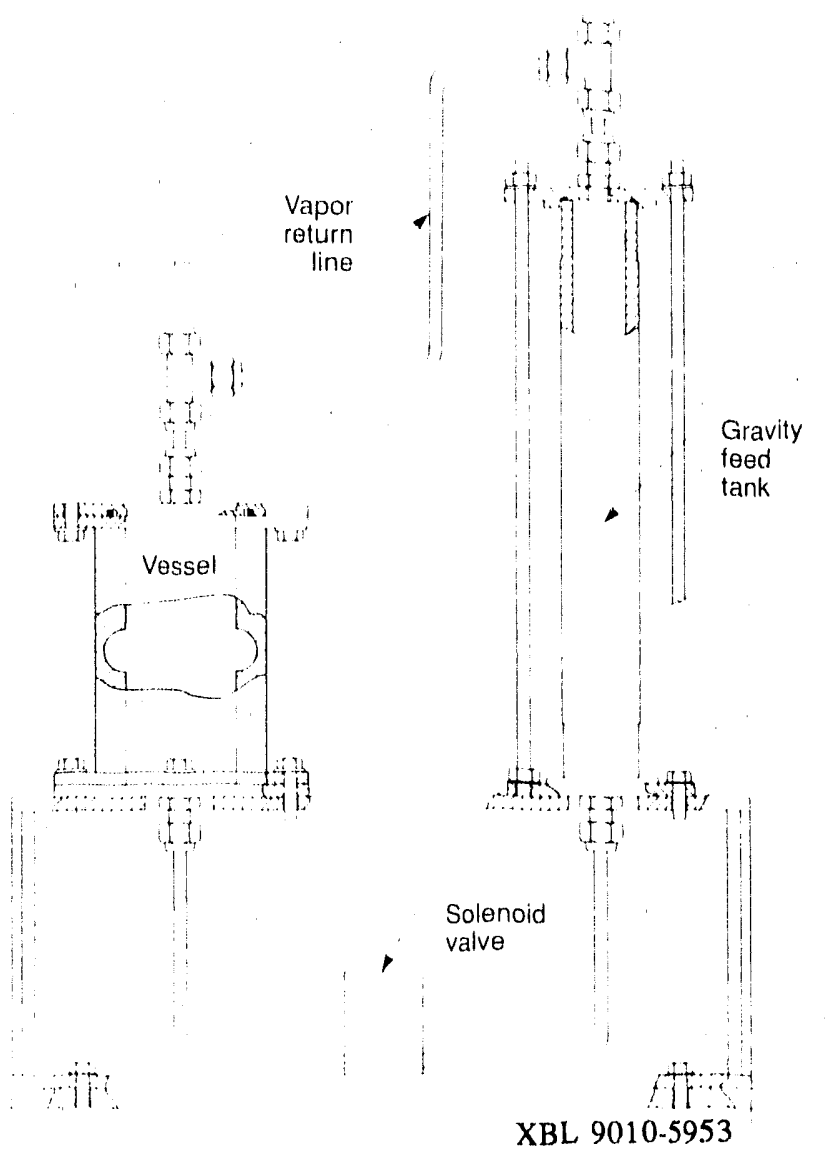

Figure 3. Schematic of experimental apparatus.

the same diameter, meeting the bottom of the bulge in a right angle. The total vessel length was approximately $12.9 \mathrm{~cm}$. All surfaces were lightly finished with cloth and a polishing compound. The interior surface after polishing and annealing deviated less than $50 \mu \mathrm{m}$ from the specified, calculated one. The vessel, its top and bottom stainless steel flanges, and other components were assembled and installed on an experimental platform suitable for the five-second drop tower at NASA Lewis Research Center.

\section{Experimental procedure}

The vessel was annealed between each drop-tower run to protect against crazing of the optical surfaces from residual/concentrated stresses caused by the machining and cleaning procedures and rapid deceleration at the bottom of the drop tower. The internal surfaces of the vessel were prepared by sequential rinses with a strong ethanol/distilled water solution and distilled water. The vessel was then allowed to air-dry in a clean-room environment.

Several procedures to determine the equilibrium contact angle of water on acrylic plastic were performed with specimens prepared in the above manner. These ircluded the standard sessile-drop tilt/slide method, a capiiiary tube method, and a wedge method. The first method placed a bound of $58^{\circ}$ to $100^{\circ}$ on the desired value by indicating the range of hysteresis for the fluid/solid pair. The capillary tube method ${ }^{1}$ was applied by machining and polishing a rather large capillary tube $(2 \mathrm{~cm}$ in diameter), partly filling it with water, and dropping it in a 2.2 second drop tower. The curvature of the equilibrium free-surface (a spherical cap in zero gravity) was measured and the results used to determine the contact angle. The value of the contact angle determined by this technique depended significantly on the initial free-surface shape, which could be varied, within the bounds of hysteresis, by small modifications of the fill procedure. The method gave values of between $67^{\circ}$ and $90^{\circ}$. The wedge method (Ref. 2, pp. 220-221) was found to give the most consistent results. It is based on the discontinuous behavior of a capillary free-surface in a wedge. If $\alpha$ is the interior half-angle of the wedge, then the surface has distinctly different behavior depending on whether the contact angle $\gamma$ is less than or greater than a critical value of $\alpha$. For a wetting liquid, if $\alpha+\gamma<90^{\circ}$ fluid climbs up the corner, if $\alpha+\gamma \geq 90^{\circ}$ it does not. In the present case $\alpha$ was varied by adjusting the angle between two contacting vertical plates of acrylic resting in a pool of distilled water. The angle $\alpha$ was decreased until the critical behavior of the fluid was observed. At this point $\alpha$ was measured and $\gamma$ calculated as $90^{\circ}-\alpha$. The technique produced contact angle values of $80^{\circ}$ for distilled water on acrylic, and the measurement was repeatable to $\pm 2^{\circ}$. These values are consistent with those reported in Ref. 2. A low-gravity refinement of the wedge method (see Ref. 5, pp. 191-192), including possible effects of hysteresis, is being tested with ground-based experiments and will be investigated in a space experiment on the International Microgravity Laboratory flight (IML-2) scheduled for 1993.

The vessel, positioned with its symmetry axis vertical, was filled in the laboratory with the prescribed liquid volume corresponding to a horizontal planar interface, which makes an $80^{\circ}$ contact angle with the container bulge. For this volume, as predicted, there was no observable curvature in the meniscus; the interface was flat. The fluid was then drawn slowly into the gravity feed tank and the solenoid valve was closed. The experimental platform containing the apparatus was then positioned in the drop tower, which was then sealed and evacuated. Prior to the release of the package the solenoid valve was actuated, permitting the fluid gradually to fill the vessel. 'This procedure was adopted to keep to a minimum the disturbances introduced to the large and sensitive free surface in filling the container. During the drop, the vessel was backlit by a diffuse white light panel and filmed with a high speed motion picture camera at 400 frames per second. The film image was subject to 


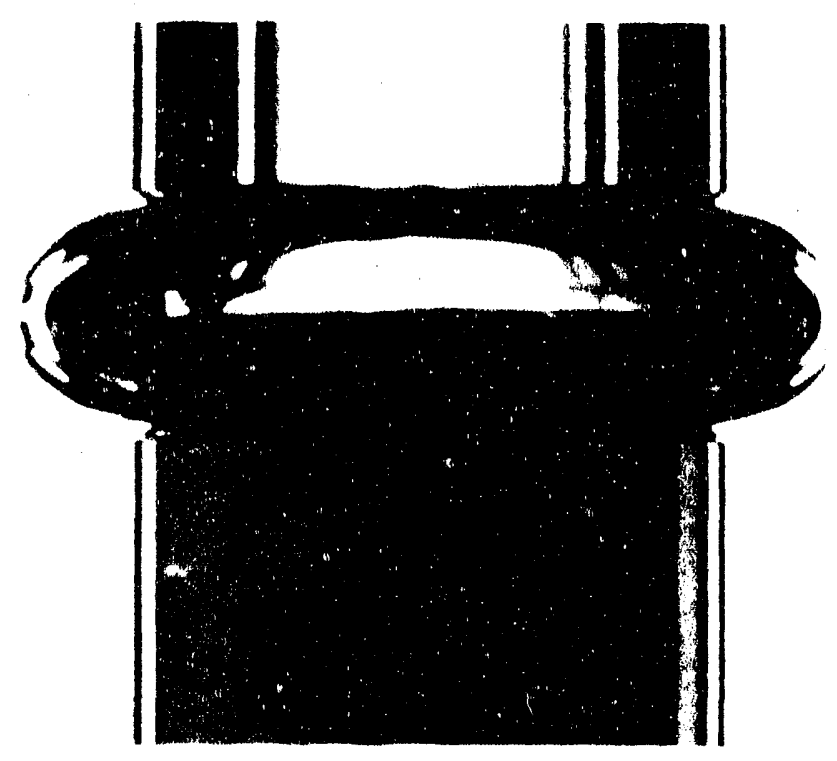

CBB 900-8661

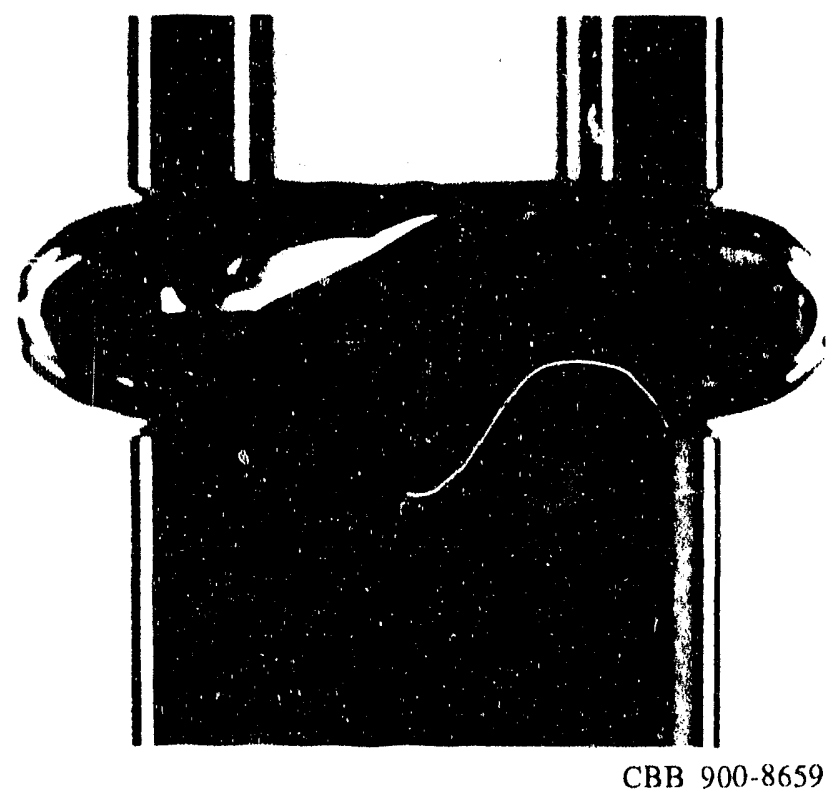

Figures 4a-b. Fluid configurations: 4a. (upper) Flat interface initial configuration; 4b. (lower) Nonsymmetric terminal configuration.

any optical distortion resulting from the mismatch in refractive index 1.33 of the distilled water and 1.49 of the acrylic plastic.

\section{Experimental results}

Several experiments were performed for observing the subsequent behavior of the initial 1-g equilibrium interface over the five-second period of free fall after release of the vessel in the drop tower. The experiment of central interest was for the vessel filled with the prescribed liquid volume for an initially flat interface, as described above. The flat interface is an equilibrium solution for any gravity level, but for zero $g$ the interface is unstable in this vessel under the idealized theory, as are all members of the symmetric equilibrium family depicted in Fig. 1. The experiment was repeated a number of times. For some cases the surface remained essentially flat, in its initial configuration (Fig. 4a). For other cases the surface reoriented to a non-symmetric one, as shown in Fig. 4b. Which situation would occur could not ise predicted in advance; it probably depended on the nature of the small perturbation imparted to the fluid at the initiation of free fall. It is significant, however, that at least in some cases the fluid did move in the five seconds of free fall from its initial flat equilibrium configuration to an obviously non-symmetric one, in accordance with the mathematical theory.

A series of experiments was carried out with the vessel tilted a small amount from vertical, in an at iempt to overcome surface friction that in some cases might be preventing surface reorientation. This procedure biased the surface toward a particular nonsymmetric surface from the outset. It was found that for initial tilts of greater than $2^{\circ}$ the non-symmetric shape of Fig. 4 b resulted, whereas for smaller tilts the behavior was again unpredictable.

Additional experiments, with under- and over-fill volumes, were conducted to indicate the sensitivity of the above results. The amounts of fluid less than, or in excess of, the precise fill level were generally in the neighborhood of $10 \mathrm{ml}$. For initially vertical vessels, the under-filled cases resulted in reorientation during free fall to a symmetric surface that domed upward in the center, while the over-filled cases resulted in a surface that domed downward. None of the cases reoriented to a non-symmetric interface, such as in Fig. 4b. Twc over-filled cases with initial tilt were tested. For one with a $5^{\circ}$ tilt a non-symmetric surface did develop. For the other, with a $1^{\circ}$ tilt (and only about a $3 \mathrm{ml}$ overfill), although an apparently stable "domed-down" meniscus was formed, it remained "offaxis".

The off-axis "sticking" phenomenon was investigated for similar tests performed on right circular cylinders for the larger contact angle fluid/solid pairs (i. e., with wetting angles substantially closer to $90^{\circ}$ than to $0^{\circ}$ ), such as water and acrylic. For these larger values of contact angle an initial tilt resulted in a nonuniform initial angle of contact at the contact curve and an initial non-rotationally-symmetric surface configuration. (Typically, contact-angle hysteresis for wetting liquids is more prominent at larger contact angles.) After a cylinder was smoothly introduced into free fall, the asymmety of the initial configuration persisted. For large initial tilts of sufficient amplitude, the ensuing motion resulted in the contact line beconing "un-stuck" and the surface reorienting toward the axially symmetric minimum energy state predicted by the Young-Laplace theory. 


\section{Acknowledgment}

This work was supported in part by the National Aeronautics and Space Administration under Grant NAG3-1143, by the National Science Foundation under Grant DMS89-02831, and by the Mathematical Sciences Subprogram of the Office of Energy Research, U.S. Department of Energy, under Contract Number DE-AC03-76SF00098.

\section{References}

${ }^{1}$ A. Chai, Accurate measurement of contact angles, AIAA 27th Aerospace Sciences meeting, Reno, NV, Jan. 1989.

${ }^{2} \mathrm{P}$. Concus and R. Finn, On capillary free surfaces in a gravitational field, Acta Math. 132 (1974), pp. 207-223.

${ }^{3} \mathrm{P}$. Concus and R. Finn, Instability of certain capillary surfaces, Manuscr. Math. 63 (1989), pp. 209213.

${ }^{4} \mathrm{P}$. Concus and R. Finn, Exotic containers for capillary surfaces, J. Fluid Mech., to appear.

${ }^{5} \mathrm{P}$. Concus and R. Finn, ('apillary surfaces in microgravity, in Low-Gravity Fluid Dynamics and Transport Phenomena, J. N. Koster and R. L. Sani, eds., Progress in Astronautics and Aeronautics, Vol. 130, AIAA, Washington, DC, 1990, pp. 183-206.

${ }^{6} \mathrm{R}$. Finn, Equilibrium Capillary Surfaces, Springer-Verlag, New York, 1986.

${ }^{7} \mathrm{R}$. Finn, Nonuniqueness and uniqueness of capillary surfaces, Manuscr. Math. 61 (1988), pp. 347-372. 

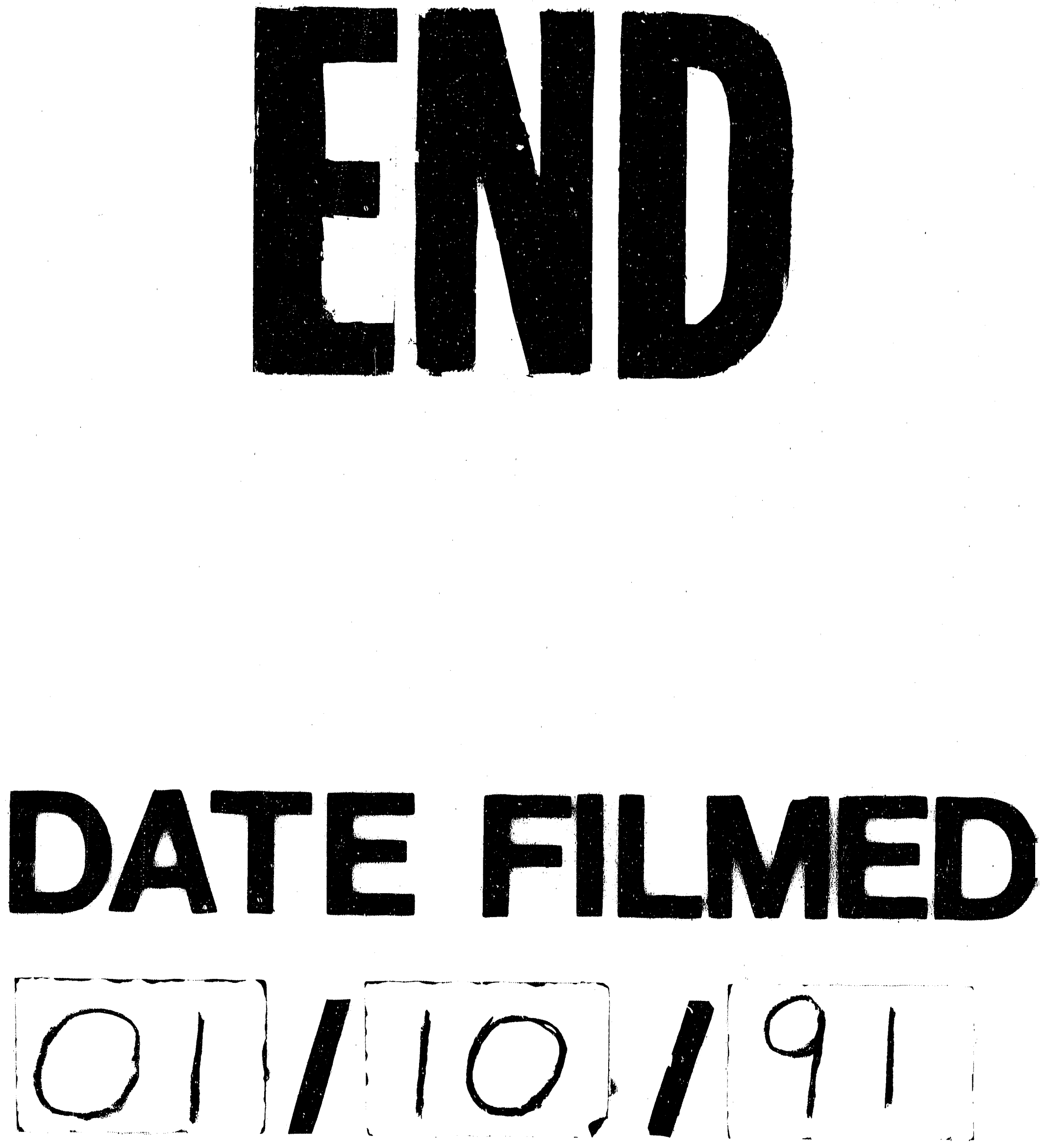
